\title{
Téoros
}

Revue de recherche en tourisme

\section{Les stratégies concurrentielles des stations de ski} Une perspective nord-américaine

\section{Vincent Sabourin}

Volume 17, numéro 3, automne 1998

Pratiques contemporaines de gestion dans le domaine du tourisme

URI : https://id.erudit.org/iderudit/1072164ar

DOI : https://doi.org/10.7202/1072164ar

Aller au sommaire du numéro

Éditeur(s)

Université du Québec à Montréal

ISSN

0712-8657 (imprimé)

1923-2705 (numérique)

Découvrir la revue

Citer cet article

Sabourin, V. (1998). Les stratégies concurrentielles des stations de ski : une perspective nord-américaine. Téoros, 17(3), 26-29.

https://doi.org/10.7202/1072164ar d'utilisation que vous pouvez consulter en ligne.

https://apropos.erudit.org/fr/usagers/politique-dutilisation/ 


\section{LeS STRATÉGIES CONCURRENTIELLES DES STATIONS DE SKI}

UNE PERSPECTIVE NORD-AMÉRICAINE

\section{Vincent Sabourin}

Dans le domaine du tourisme, on assiste depuis quelques années à l'émergence du paradigme de la gestion stratégique. Ce paradigme identifie les facteurs qui sont sous le contrôle de la gestion de l'entreprise touristique et qui peuvent l'anener à influencer considérablement les phénomènes de marché et de concurrence. Le présent article vise à illustrer une application des concepts de gestion stratégique en prenant l'exemple des récents développements dans l'industrie nord-américaine des stations de ski.

De façon plus spécifique, cet article vise les objectifs suivants :

- Illustrer l'application des concepts de gestion stratégique afin de guider le gestionnaire de l'entreprise touristique.

- Mettre en évidence les stratégies concurrentielles ayant un caractère générique dans l'industrie touristique.

- Illustrer le phénomène général de l'industrialisation des services touristiques en prenant l'exemple de la stratégie d'Intra-West.

\section{MÉTHODOLOGIE}

La méthode utilisée repose sur le modèle de gestion stratégique découlant principalement de l'économie industrielle. De façon particulière, notre analyse s'appuie sur les modèles de Scherer (1980) et de Porter (1980).

\section{LE MODËLE \\ DE L'ANALYSE INDUSTRIELLE}

D'une part, le modèle de l'analyse industrielle met en évidence le rôle joué par le contexte stratégique sur la structure d'une industrie. D'autre part, il illustre comment la structure de l'industrie vient, à son tour. influencer les stratégies des entreprises et leur performance. Le modèle d'analyse industrielle rassemble quatre groupes de facteurs interreliés :

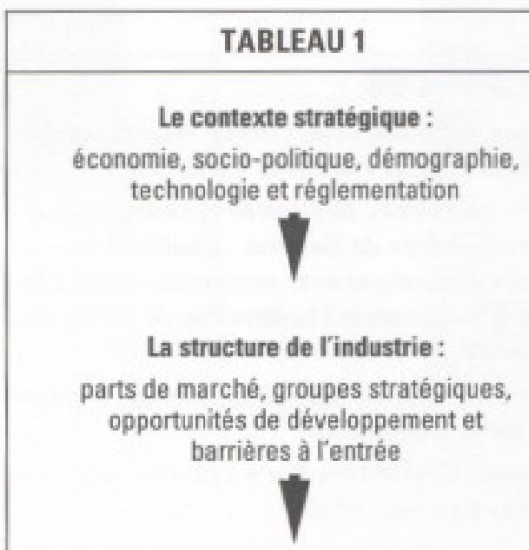

Les stratégies concurrentielles :

comportements stratégiques des entreprises sur le plan du marketing.

de la production et de la finance

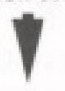

La performance des entreprises :

le nombre d'entreprises, leur rentabilité, le profil des entreprises gagnantes et perdantes

\section{LE MODEELE DES STADES D'ÉVOLUTION DE L'INDUSTRIE}

Nous avons aussi utilisé le modèle de Porter (1980), soit celui des stades d'évolution d'une industrie, afin de saisir sa dynamique. Toute industrie traverse quatre stades de développement : l'émergence, la croissance, la consolidation et la maturité. Chaque stade comporte un certain nombre de caractéristiques particulières. Plus l'industrie est jeune et plus l'entrée se fait facilement. Plus elle atteint la maturité et plus les investissements pour y entrer deviennent importants. Lorsqu'une entreprise atteint le stade de la maturité, les taux de croissance du marché chutent, des marques de commerce apparaissent et de grandes entreprises bénéficiant d'économies d'échelle viennent dominer le marché.

\section{LA RECHERCHE D'INFORMATION}

Nous avons réalisé notre recherche à partir des données secondaires recueillies lors d'une recherche documentaire sur l' industrie nord-américaine des stations de ski. Nous avons ainsi recueilli et analysé environ une vingtaine de sources d'information afin de mettre en évidence les stratégies concurrentielles de cette industrie.

\section{L'ANALYSE DES RÉSULTATS}

\section{LE CONTEXTE STRATÉGIQUE DE L'INDUSTRIE}

Quels sont les facteurs qui expliquent les stratégies concurrentielles des stations de ski ? Parmi les différents facteurs, se re- 
trouvent les changements démographiques et la chute du taux de la croissance du marché.

On trouve tout d'abord un facteur démographique lié au vieillissement de la population. Dans les années $1960,70 \%$ des skieurs avaient moins de 35 ans. Dans les années 1990, ce nombre est tombé à seulement $50 \%$. Le vieillissement de la population a amené une diminution signifi= cative de l'intensité des activités des skieurs, d'une part, mais aussi une transformation des besoins des consommateurs. Ainsi, le vieillissement de la population et la proportion accrue des 35 ans et plus sur les pentes de ski, a favorisé les stations de ski qui ont fait l'objet d'investissements importants. Ce vieillissement de la population a des tendances négatives sur les petites stations de ski et sur les stations de taille intermédiaire. Une entreprise comme Key Stone a maintenant trois stations de ski qui totalisent 2,5 millions de skieurs par jour alors qu'une compagnie comme Ski Limited bénéficie de plus de 1,9 millions de ski-journées.

Un second facteur lié au contexte stratégique est le phénomène du cycle de vie du marché. Le marché des stations de ski a connu une forte croissance et a permis l'établissement d'un nombre important de nouvelles stations de ski. L'offre s"est accrue mais la croissance a ralenti. Ainsi, l'offre est maintenant supérieure à la demande et cela a des conséquences importantes. Ce contexte met en place des conditions qui faciliteront une épuration du secteur.

Le marché du ski approche de la maturité. Cela signifie que, pour poursuivre leur croissance, les stations de ski doivent maintenant attirer des clients de leurs concurrents ou encore développer des stratégies agressives afin de rejoindre de nouveaux adeptes du ski. Dans les deux cas, ces stratégies sont coûteuses et nécessitent des investissements plus importants qu'auparavant. Un marché à maturité signifie aussi des stratégies plus coûteuses qui viennent diminuer considérablement la marge brute des stations de ski.

Le contexte stratégique des stations de ski est aussi très particulier puisque les stations doivent composer avec des exigences environnementales beaucoup plus grandes qu'auparavant. De plus, les revenus de ce secteur demeurent tributaires des variations saisonnières et ce, malgré les investissements effectués dans les canons à neige. Un hiver avec peu ou pas de neige peut signifier que $60 \%$ des stations de ski nord-américaines feront peu ou pas de profit.

\section{LA STRUCTURE DE L'INDUSTRIE}

Quels sont les facteurs liés à la structure de l'industrie qui expliquent les stratégies des stations de ski en Amérique du Nord ? Parmi les facteurs qui expliquent la vague de fusions et d'acquisitions vécue dans l'industrie, il y en a qui sont liés au fait que l'offre est maintenant supérieure à la demande. De plus, les exigences en matière de capitalisation sont accrues et ce, à différents niveaux. Les investissements des stations de ski, sous la forme de programmes de publicité et de formules d'abonnement (comme la tarification à l'heure) afin de fidéliser la clientèle, exigent des déboursés très importants et affectent les marges bénéficiaires.

Les investissements dans les produits touristiques se traduisent aussi par le développement de marques de commerce. De plus en plus les stations de ski doivent investir des montants considérables afin d'accroître leur notoriété auprès des consommateurs.

Ces exigences accrues sur le plan de la capitalisation se manifestent aussi par des stratégies de développement immobilier comme des projets de construction domiciliaire et hôtelier afin de s' accaparer une part plus grande qu'auparavant des revenus associés à l'activité du ski. Le développement immobilier d'Intra-West au Québec illustre ce phénomène.

Afin de stabiliser leur revenus, les stations de ski doivent investir dans des canons à neige de façon à minimiser les perturbations climatiques de leurs activités. Elles doivent aussi investir dans des remontepentes mécanisés à haute vitesse de façon à satisfaire les exigences des skieurs en période de pointe.

Ces exigences importantes de capitalisation ont des effets structurants sur l'industrie des stations de ski et engendrent une épuration du nombre de stations. Alors que le nombre de visiteurs par jour est demeuré stable à 50 millions au cours des 20 dernières années, les exigences sur le plan des investissements ont coûté à l'industrie du ski plus de 2,3 milliards de dollars.

La capitalisation accrue a des conséquences sur la structure industrielle du secteur. Elle correspond à un phénomène général d'industrialisation. L' industrie est de plus en plus structurée avec des méga-centres ; un nombre de plus en plus restreint de petits centres desservent des marchés locaux. En outre, le marché a de plus en plus de segments, ce qui a pour effet de diminuer le caractère « substituable $*$ des activités de ski. Un adepte du Mont-Tremblant ne fréquentera pas les stations orientées famille ou encore une station locale avec des pentes peu attrayantes pour lui.

La capitalisation importante d'un secteur jusqu'à présent relativement fragmenté et moins industrialisé que des secteurs touristiques, comme les lignes aériennes ou les chaînes hôtelières, représente maintenant une formidable barrière à l'entrée de nouveaux joueurs. Elle augmente de façon considérable la mise de fonds et les coûts récupérables pour pénétrer le secteur et s'accaparer une part de marché.

Sur le plan économique, cette industrialisation est associée à la naissance croissante d'économie d'échelle et d'un seuil minimal d'efficacité important. Les stations de ski de plus grande dimension sont en mesure d"amortir plus facilement leurs coûts que les petites stations locales dont les opérations sont de moindre importance.

Ainsi un remonte-pente à haute vitesse peut couter plus de 2 millions de dollars. La station de ski Black Comb est maintenant capable de transporter jusqu'à 22400 skieurs à l'heure ; cette station appartient à Intra-West. Les remonte-pentes, les dix restaurants ainsi que les autres développements ont coûté à Intra-West plus de 60 millions de dollars en 86 .

Fait relativement nouveau, cette industrialisation est maintenant aussi associée à un phénomène d'économie d'envergure. Les économies d'envergure proviennent des coûts partagés par une entreprise qui possède plusieurs stations de ski. Elles représentent un phénomène de concurrence important pour les stations indépendantes qui doivent assumer la totalité des coûts.

Ainsi les stations de ski ayant à leur actif plusieurs pentes et des localisations différentes sont en mesure de baisser le 
coût unitaire et le prix de revient d' un billet de ski. Elles créent des économies d'envergure géographique. Ces économies d'envergure se manifestent aussi sur le plan commercial ; ainsi la compagnie Key Stone est en mesure de faire des promotions. croisées pour l'ensemble de ses stations de ski afin d'accroître les bénéfices de la valeur d'un billet auprès des consommateurs. Les principaux joueurs de l'industrie sont donc à la recherche d'une synergie corporative qui leur permet de partager leurs coûts d'opération sur le plan administratif, sur le plan de l'entretien des stations de ski et sur le plan commercial. Enfin, on trouve aussi des économies d'envergure de segment puisque qu'une entreprise pourra joindre des segments différents à l'intérieur d'une même opération. Par exemple, Intra-West joint à Tremblant le segment ski en hiver et le segment tennis ou golf en été. Des segments loisirs différents, mais des coûts de publicité et de promotion partagés sur la base de la même clientèle.

\section{LES STRATÉGIES CONCURRENTIELLES DE MARCHÉ}

Dans ce contexte, la consolidation de la structure industrielle a des conséquences importantes sur le comportement strategique et les stratégies concurrentielles des stations de ski. Les stratégies de domination du marché apportent une forte concurrence parmi les principaux joueurs de l'industrie: Resort Management, Ski Limited, Tamovi Temto, Vail Associated, Aspen Skiing et la compagnie canadienne Intra-West qui a récemment fait l'acquisition de Mont-Tremblant.

L'examen des stratégies des quatre principaux joueurs dans le domaine des stations de ski montre des comportements misant avant tout sur le développement d'avantages concurrentiels particuliers. Ces avantages sont le résultat de stratégies d'acquisitions permettant une diversification géographique des activités. La diversification géographique est devenue essentielle afin de stabiliser les cash-flows, de répondre aux exigences accrues des actionnaires et d'offrir des rendements stables et peu sensibles a la conjoncture climatique. Ainsi, le fait qu' Intra-West ait fait l'acquisition de Tremblant l'a mis en partie à l'abri de la récession qu'a connue la Colombie-Britannique au cour's des derniers mois.
La diversification géographique permet donc de mettre les stations de ski à l'abri des variations saisonnières ou des conjonctures économiques dans un marché régional donne.

On rencontre aussi comme comportement stratégique la diversification saisonnière. Les principaux joueurs dans les stations de ski ont fait des investissements importants afin de desaisonnaliser leurs sources de revenus. Ainsi, pour se mettre à l'abri d'une mauvaise saison de ski, ils ont fait des investissements, notamment dans des terrains de golf et des courts de tennis de façon à être en mesure d'accroître la proportion de leurs revenus provenant de l'autre saison.

Des joueurs comme Intra-West ont fait des investissements importants de façon à segmenter leur marche. Intra-West se spécialise aussi dans le segment des babyboomers et mise sur les consommateurs qui auront bientôt fini de rembourser l'hypothèque de leur maison et pourront prendre le chemin de la montagne en quête d'une résidence secondaire. Intra-West a donc utilisé à son avantage les changements démographiques en développant un style de vie et en ajoutant de la plus-value à ses sites. Par des investissements consacrés à la construction de complexes hôteliers et commerciaux qui visent à retenir la clientèle et par l'érection de copropriétés de style champêtre au beau milieu de ces sites, Intra-West est en mesure d'obtenir des revenus stables provenant de visites régulières de ses consommateurs. Lorsque l'entreprise a acheté la station du Mont-Tremblant en 1991, 380000 personnes fréquentaient le centre de ski en hiver. Sept ans et des centaines de millions de dollars plus tard, le site accueille 800000 personnes l'hiver et 1,2 millions l'été. Cela fait de Tremblant l'une des stations les plus achalandées en Amérique du Nord. De façon à " désaisonnaliser " ses revenus, Intra-West a fait l'acquisition de Raven Golf Group en aménageant des terrains à proximité des villages alpins. C'est cette stratégie qui a valu à Intra-West d'etre classée numéro un en Amérique du Nord, selon la revue Ski Magazine, comme le meilleur gestionnaire dans sa catégorie.

La stratégie de chacun des principaux joueurs est de développer une masse critique de skieurs et de visiteurs qui lui permette d'amortir ses investissements. Cette masse critique permet entre autres de diminuer considérablement le coût des achats de publicité puisque les stations de ski disposent maintenant d'un plus grand nombre de pentes.

La stratégie des stations de ski est donc de transformer les actifs économiques tangibles que sont les pentes en actifs économiques intangibles et de transformer les stations de ski en destinations plaisir et vacances. Une station comme Saint-Sauveur bénéficie déjà d'un actif intangible sur le plan de l'image. Le fabricant de voiture BMW qui commandite plusieurs stations de ski a compris ce phénomène et cherche à bénéficier du positionnement des stations, puisqu'il correspond à celui de l'acheteur potentiel de voitures à la conduite sportive comme celle de BMW.

Afin de dominer leur marché ces entreprises ont développé des stratégies de commercialisation bien ciblées, mais aussi des systèmes stratégiques perfectionnés de contrôle des coûts. Ainsi la compagnie Ski Limited a mis en place une véritable culture corporative du contrôle des coûts. Elle a investi dans des ordinateurs et des systèmes de prix de revient permettant le suivi détaillé de chacune des activités de l'entreprise et de prévoir avec précision les changements climatiques. Le système remettra à l'opérateur d'une boutique de ski un rapport mensuel détaillé de ses activités et de sa rentabilité.

L'évaluation détaillée du système de coût a aussi permis de mettre en place un système de prévision perfectionné et d'en évaluer les résultats sur une base régulière. Les systèmes de coût permettent une meilleure gestion des ressources humaines, par exemple en postant le mềme employé comme préposé au stationnement le matin lorsque le skieur arrive et au service alimentaire a l'heure du lunch.

Enfin, une stratégie agressive d'acquisitions qui vise le développement des actifs intangibles dans des produits que sont les stations de ski, nécessite des investissements dans des sites géographiques de première qualité. C'est pourquoi la localisation commerciale des stations de ski revêt une telle importance. Ainsi la station de Whistler connaît un grand succès et une forte rentabilité notamment parce que cette station est à 8 heures de vol direct du Japon sans escale et que le taux de change avantageux du dollar canadien permet d'offrir aux voyageurs japonais des vacances de 
grande valeur à un prix imbattable en Amérique du Nord. Les stratégies d'acquisition reposent donc sur l'achat d'un site géographique avec un bassin de skieurs à proximité dont le potentiel de développement est important. Ce fut le cas du MontTremblant lors de l'acquisition par IntraWest.

\section{LA PERFORMANCE}

Les stratégies concurrentielles ont plusieurs conséquences et amènent une vague de fusions et d'acquisitions. La vague de fusions et d'acquisitions amène une épuration progressive de l'industrie et force les joueurs peu performants à fusionner ou à devenir la cible d'acquisition de stations plus performantes.

Depuis le début des années 1990, l'industrie nord-américaine des stations de ski vit une période de consolidation qui se manifeste par de nombreuses fusions-acquisitions, des alliances stratégiques, des émissions publiques d'actions, des opérations de rachat d'actions, des restructurations. Toutes ces initiatives visent en premier lieu à assurer la rentabilité des stations de ski et à accroître le rendement des actionnaires. Ainsi depuis le début des années 1980 , le nombre de stations de ski indépendantes aux États-Unis a diminué de plus de $30 \%$. Durant la saison 19791980 , il y avait 845 stations de ski exploitées aux États-Unis ; en 1992-1993, il n'y en a plus que 529 et la tendance s'accentue.
Les principaux joueurs américains sont entrés dans des phases d'acquisitions.

Selon une enquête auprès de l'industrie américaine, les marges des stations de ski ont aussi diminué de façon importante au cours des dernières années et ne sont que de $2,8 \%$. Elles étaient de $12,4 \%$ avant intérêt et dépréciation auparavant.

\section{CONCLUSION}

L'analyse de la consolidation du secteur des stations de ski en Amérique du Nord met en lumière l'industrialisation à laquelle font face les entreprises du secteur touristique. Elle montre comment les impératifs économiques du marché sont en train de transformer graduellement la stratégie des differentes stations de ski. Elle montre aussi comment la stratégie des stations de ski, notamment celle d'Intra-West, repose sur une valorisation de la ressource touristique, d'une part, mais aussi sur des stratégies agressives de commercialisation permettant de mettre en valeur la ressource.

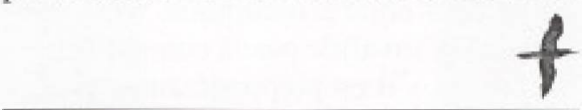

\section{RÉFÉRENCES}

Minka, Lina (1994), * Perfect Partners *, Revue Ford, 23 mail.

Business Week (1996), * The Club Med of the Ski Slopes? mars, p. 64-66.

Canadian Share Owners (1997), * Intra West Corporation $\cdots$, janvier-fevrier.
Le Devoir (1998), * Intra West Internautes, un autre centre $\%$, mardi 10 février, p. B-2.

Fontaine, Laura (1998), * Avalanche sur MontTremblant *, Commerce, janvier.

McCune, Jenny (1994), * Downhill Pathole : Ski Resorts Fight for Survival w, Management Review, tévrier.

Cumming, Rob (1995), \& Snow Job s, Journal of Business Strategy, janvier-février, p. 30-36.

Lane, Randall (1996), * Check Out in Skiing *, Forbes 6 mai.

Europe $(1997)$, \& Snow à la carte *, février. p. 33-34.

Business Week (1993), wSkiing : During the Whole Family List $*$, Business Week, 18 octobre.

Business Week (1993), * Sking : On the Acquisition Trail $\%, 6$ decembre.

The Corporate Growth Report (1993), * Ski Resort Companies Consolidated $*, 6$ décembre.

Business Week (1995), a Going to Extreme to Lure the Hot Dog Back w, Business Week, 13 mars, p. 100.

Colorado, Vail (1998), « Winter Wonder Lands \&, The Economist, 31 janvier.

Germain, Daniel (1998), * Intra West : A la conquête de nouveaux sommets \%, Les Affaires, 5 mars, p. 67. 